\title{
Integrated Scheduling System on Steel-Making and Continuous Casting with Application ${ }^{1}$
}

\author{
Kebin Lu*, Kewei Huang* and Dingwei Wang** \\ *.Institute of Automation, Baosteel Group, Shanghai,201900, P.R.China \\ (Tel: +86(21)26643468,e-mail: \{Lukb,kwhuang\}@baosteel.com) \\ **. Information School, Northeastern University, Shenyang, 110004, P.R.China \\ (Tel: +86(24)83680662,e-mail:dwwang@mail.neu.edu.cn)
}

\begin{abstract}
The integrated scheduling problem of steel-making and continuous casting is discussed. To solve the problem we develop a scheduling system with the architecture of three tiers. Its main functions include: schedule making and adjusting, matching and optimization, abnormal logistics handling and production information management. A hybrid heuristic and optimization algorithm is developed for the solution. The scheduling system had been embedded into the whole MES of a big iron and steel group in China. This approach had been applied to a branch plant of stainless steel. The satisfactory results have been achieved.
\end{abstract}

\section{INTRODUCTION}

The integrated scheduling optimization of steel-making and continuous casting can efficiently enhance productivity and serve energy cost (Pacciarelli and Pranzo 2004). The steel making and casting systems usually consist of many kinds of automatic equipments (Craiga et al 2001). The production and scheduling are very complex problems. Therefore, the research on the methodology of integrated scheduling has been a very active area (Tang et al 2001). Mathematical programming, mix integer programming and multi-level decomposition approach were reported in literatures (Tang et al 2000, Bellabdaoui and Teghem 2006, Harjunkoski and Grossmann 2001).

The studied steel making and casting system of a stainless steel plant consists of two parallel converters and two parallel conticasters. There are three refining furnaces between the converters and conticasters. The all feasible integrated schedules are subject to weekly production planning, the technique requirements and constraints of equipment capacity and energy limitations. The objective of the scheduling is to shorten the make span of steel-making and casting and enhance total throughput of the system without any equipment conflicting.

To solve the practical scheduling problem, we develop a scheduling software package with architecture of three tiers. A heuristic and optimization hybrid algorithm is embedded as backbone of the system. This approach had been applied to a branch plant of stainless steel. The satisfactory results have been achieved.

\footnotetext{
${ }^{1}$ The research was partly supported by the National Natural Science Foundation of China (No. 70431003, 60521003) and the National Science Support Program (No. 2006BAH02A09).
}

\section{ARCHITECHURE OF INTEGRETED SCHEDULING SYSTEM}

The scheduling system is a typical Client/Server architecture of three-tier. The server is installed with HP UNIX system with the database of ORACLE 9.0. The middleware is TUXEDO. The clients are developed by Visual Basic, which use ActiveX technology and can easily be deployed in distributed terminals. For safe, User certification technology is integrated into Application. The system architecture is shown in Fig.1.

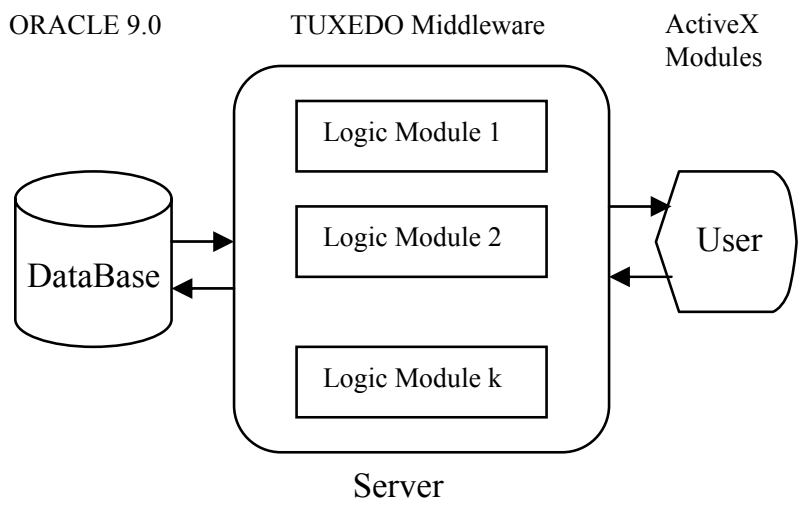

Fig. 1. The system architecture of three levels

The features of the system include:

1) The three-tier architecture of system makes that all terminals can reach the same consistent and simultaneous scheduling results. The user certification can guarantee the security of the computer system. 
2) The system has a friendly interactive HMI(HumanMachine Interface). Because there usually are many special situations in Steel-Making production cannot be done by computer only, the HMI can provide feasible solution for operators to process all kind of complex and emergent cases.

3) The scheduling system is embedded into the MES (Manufacturing Executive System) of the steel works. Thus, all information of weekly/daily production plan, customer orders, technique requirements and equipment capacity can be obtained from MES.

\section{FUNCTION MODULE DESIGN}

The main functions include:

1) Schedule Making and Adjusting: According to the weekly or daily production plan, the module makes the initial schedule subject to the technique requirement and equipment availability. The schedule sheets and Gantt Charts of all operations are generated by the module also.

2) Matching and Optimization: This module is to match the customer orders to casting sequence and equipments. With the changes of production situation the reschedule and adjustment are done by the optimization model to best match customer requirements.

3) Abnormal Logistics Handling: Since the environment of high temperature and continuous production in steel works, the production status changes and emergent events cannot be voided. The module is used to treat the abnormal logistics and rescheduling in any cases. It is done by a human-computer interactive mode of the system.

4) Production Information Management: As a part of MES, the scheduling system has to report its scheduling results and daily production records to upper level computer. Thus, the information of scheduling can integrated with whole MES system of the steel works.

\section{HEURISTIC AND OPTIMIZATION ALGORITHM}

The backbone module of the scheduling system is the hybrid heuristic and optimization algorithm. The heuristic algorithm is used to create the initial scheduling solution. The scheduling rules are generated from the experienced schedulers in practice. The heuristic can greatly speed up the computation process even it cannot guarantee the optimality. Then, a heuristic optimization method guided by expert knowledge is used to optimize the scheduling results.

The flow chart of the hybrid algorithm is shown in Fig. 2.

\section{CONCLUSIONS}

The presented integrated scheduling system with three-tier architecture has successfully applied to a stainless steel plant.
The practical application results have proved that the designed system structure and function modules are reasonable to practical applications. The hybrid heuristic and optimization algorithm can efficiently improve the results of scheduling and meet various schedule requirements.

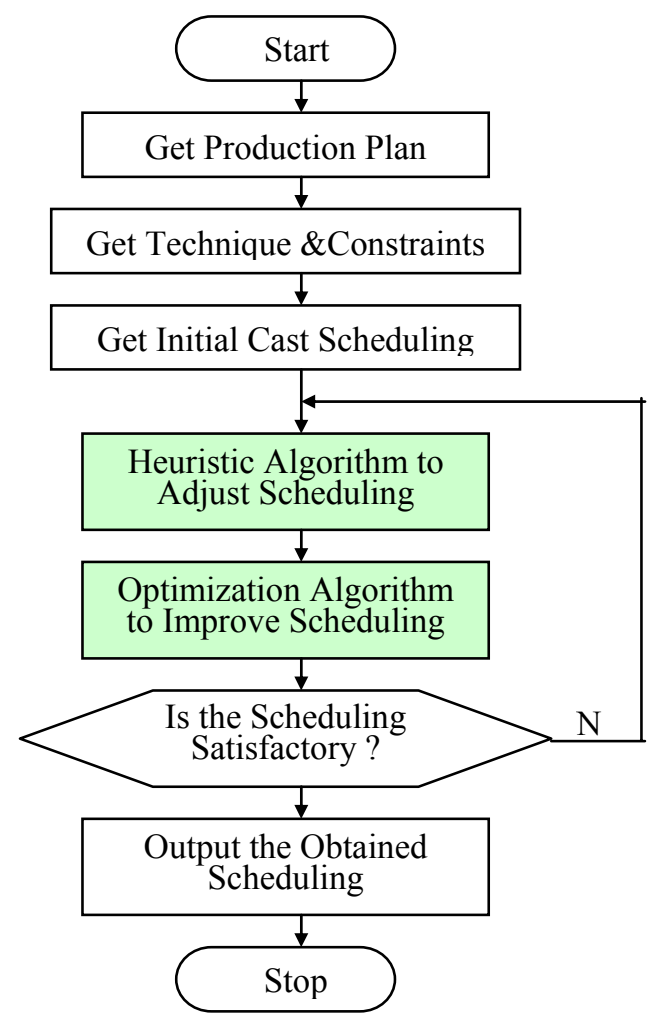

Fig.2. The flow chart of the hybrid algorithm

\section{REFERENCES}

Bellabdaoui, A. and J. Teghem (2006), A mixed-integer linear programming model for the continuous casting planning, Int. J. Production Economics, 104, 260-270.

Craiga, I.K., F.R. Camisani-Calzolaria and P.C. Pistorius (2001), A contemplative stance on the automation of continuous casting in steel processing, Control Engineering Practice, 9, 1013-1020.

Harjunkoski, I. and I. E. Grossmann (2001), A decomposition approach for the scheduling of a steel plant Production, Computers and Chemical Engineering, 5, 647-1660.

Pacciarelli, D. and M. Pranzo (2004), Production scheduling in a steelmaking-continuous casting plant, Computers and Chemical Engineering, 28, 2823-2835.

Tang, L., J. Liu, A. Rong and Z. Yang (2001), A review of planning and scheduling systems and methods for integrated steel production, Eur. J. of Operational Research, 133, 1-20.

Tang, L., J. Liu and Z. Yang (2000), A mathematical programming model for scheduling steelmakingcontinuous casting production, Eur. J. of Operational Research, 120, 423-435. 